TRANSACTIONS OF THE

AMERICAN MATHEMATICAL SOCIETY

Volume 358, Number 1, Pages 233-249

S 0002-9947(05)03651-2

Article electronically published on February 4, 2005

\title{
REAL AND COMPLEX EARTHQUAKES
}

\author{
DRAGOMIR ŠARIĆ
}

\begin{abstract}
We consider (real) earthquakes and, by their extensions, complex earthquakes of the hyperbolic plane $\mathbb{H}^{2}$. We show that an earthquake restricted to the boundary $S^{1}$ of $\mathbb{H}^{2}$ is a quasisymmetric map if and only if its earthquake measure is bounded. Multiplying an earthquake measure by a positive parameter we obtain an earthquake path. Consequently, an earthquake path with a bounded measure is a path in the universal Teichmüller space. We extend the real parameter for a bounded earthquake into the complex parameter with small imaginary part. Such obtained complex earthquake (or bending) is holomorphic in the parameter. Moreover, the restrictions to $S^{1}$ of a bending with complex parameter of small imaginary part is a holomorphic motion of $S^{1}$ in the complex plane. In particular, a real earthquake path with bounded earthquake measure is analytic in its parameter.
\end{abstract}

\section{INTRODUCTION}

Earthquakes are maps which change hyperbolic metrics on a surface. Thurston 13 introduced earthquakes on closed surfaces as a certain completion of "continuous" left Dehn twists. He showed that any two hyperbolic metrics on a closed surface are related by a unique earthquake. This fact is referred to as the earthquake theorem. An earthquake is uniquely determined by a measured lamination called an earthquake measure. Multiplying the earthquake measure by a positive parameter we get an earthquake path in the Teichmüller space of the initial surface. Kerckhoff 14] showed that an earthquake path, for a closed surface, is analytic in its parameter.

In a subsequent paper [19, Thurston gave a direct proof of the earthquake theorem for the more general case. He defined earthquakes on the hyperbolic plane $\mathbb{H}^{2}$ and showed that any homeomorphism of the boundary $S^{1}$ of $\mathbb{H}^{2}$ can be obtained by continuously extending a unique earthquake of $\mathbb{H}^{2}$ to $S^{1}$ (geology is transitive). The original earthquake theorem for compact surfaces is obtained easily from geology is transitive.

Thurston's work on earthquakes in the hyperbolic plane $\mathbb{H}^{2}$ opened the possibility of using earthquakes on infinite area hyperbolic surfaces. In particular, earthquakes could be used in Teichmüller spaces of infinite surfaces. An earthquake on a hyperbolic surface of infinite area gives another hyperbolic structure which is not necessarily quasiconformally equivalent to the initial structure. This

Received by the editors March 1, 2003 and, in revised form, February 1, 2004.

2000 Mathematics Subject Classification. Primary 30F60, 30F45, 32H02, 32G05; Secondary $30 \mathrm{C} 62$.

Key words and phrases. Earthquake, transverse measure, bending. 
paper investigates which earthquakes stay in the quasiconformal class of the initial surface and how these earthquakes depend on the parameter.

The Teichmüller space of the hyperbolic plane $\mathbb{H}^{2}$ is identified with the space of quasisymmetric maps of $S^{1}$ modulo post-composition with Möbius maps. Thus, our goal is to identify earthquakes whose continuous extensions to $S^{1}$ are quasisymmetric maps. An earthquake is uniquely determined, up to post-composition with a Möbius map, by a measured lamination which is called an earthquake measure. If we multiply an earthquake measure $\lambda$ with a positive parameter $t$, we obtain a family of earthquake measures $t \lambda$. The corresponding family of earthquakes is called an earthquake path with the parameter $t$. To talk about earthquake paths in the Teichmüller space, we need to show that if an earthquake with the earthquake measure $\lambda$ extends to a quasisymmetric map of $S^{1}$, then the whole earthquake path with earthquake measures $t \lambda, t>0$, consists of quasisymmetric maps of $S^{1}$. The following theorem characterizes earthquake maps which give quasisymmetric maps in terms of their earthquake measures.

Theorem 1. Let $E^{\lambda}$ be an earthquake of $\mathbb{H}^{2}$ with earthquake measure $\lambda$. Then the continuous extension of $E^{\lambda}$ to $S^{1}$ is quasisymmetric if and only if $\lambda$ is bounded.

An earthquake measure $\lambda$ is bounded if $\sup \lambda(I)<\infty$, where the supremum is over all geodesic arcs of length $1 \mathrm{in} \mathbb{H}^{2}$. It is immediate that if $\lambda$ is bounded, then $t \lambda$ is bounded. Thus $t \mapsto\left[\left.E^{t \lambda}\right|_{S^{1}}\right]$ is a path in the universal Teichmüller space $\mathcal{T}\left(\mathbb{H}^{2}\right)$. It is enough to prove Theorem 1 for the universal Teichmüller space $\mathcal{T}\left(\mathbb{H}^{2}\right)$, because an earthquake is invariant with respect to the covering group if and only if its earthquake measure is invariant. Consequently Theorem 1 holds for any hyperbolic surface.

Remark 1. One direction of the above theorem was proved by Thurston [19]. Independently of the author, a version of Theorem 1 is proved by Gardiner, Hu and Lakic [10, and by $\mathrm{Hu}$ [11].

It is natural to extend the real parameter $t>0$ to a complex parameter $\tau$. The extended map $\tau \mapsto E^{\tau \lambda}$ is called bending. We embed $\mathbb{H}^{2}$ as a vertical upper halfplane above $x$-axis for the model $\mathbb{C} \times\{t \mid t>0\}$ of hyperbolic three space $\mathbb{H}^{3}$. The bending map $E^{\tau \lambda}: \mathbb{H}^{2} \rightarrow \mathbb{H}^{3}$ is given by bending $\mathbb{H}^{2}$ according to the complex measure $\tau \lambda$ (see Epstein and Marden [5]). The map $E^{\tau \lambda}: \mathbb{H}^{2} \rightarrow \mathbb{H}^{3}$ trivially extends to the boundary of flat pieces (connected components of the complement of the geodesic lamination support of $\lambda$ ) and to the endpoints of the geodesics in the support of $\lambda$ on $S^{1}=\widehat{\mathbb{R}}$. However, we show that it extends to the whole boundary $S^{1}$ of $\mathbb{H}^{2}$. Namely, $E^{\tau \lambda}: \widehat{\mathbb{R}} \rightarrow \widehat{\mathbb{C}}$ is well defined for $\tau \in S_{\lambda}$, where $S_{\lambda}$ is a neighborhood of $\mathbb{R}$ depending on the Thurston norm $\|\lambda\|$ of $\lambda$. Without loss of generality we assume that $E^{\tau \lambda}(\infty)=\infty$.

Theorem 2. Let $\lambda$ be a bounded earthquake measure. Then the earthquake map $(x, t) \mapsto E^{t \lambda}(x), x \in \mathbb{R}$ and $t>0$, extends to a holomorphic motion $(x, \tau) \mapsto$ $E^{\tau \lambda}(x), x \in \mathbb{R}$ and $\tau \in S_{\lambda}$, of the real line.

Remark 2. In the proof of Theorem 2 we analyze the bent plane $E^{\tau \lambda}\left(\mathbb{H}^{2}\right)$ in $\mathbb{H}^{3}$. It turns out that $E^{\tau \lambda}\left(\mathbb{H}^{2}\right)$ divides $\mathbb{H}^{3}$ into two sets, one of them being convex. The convex set faces one of the two components of $\mathbb{C}-E^{\tau \lambda}(\mathbb{R})$ and the boundary of the set in $\mathbb{H}^{3}$ is $E^{\tau \lambda}\left(\mathbb{H}^{2}\right)$. Another proof of the same result has recently been obtained by Epstein, Marden and Markovic [6]. 
By a theorem of Slodkowski [18, a holomorphic motion of a closed set in $\mathbb{C}$ can be extended to a holomorphic motion of $\mathbb{C}$. Thus $(x, \tau) \mapsto E^{\tau \lambda}(x), x \in \mathbb{R}$ and $\tau \in S_{\lambda}$, extends to $(z, \tau) \mapsto E^{\tau \lambda}(z), z \in \mathbb{C}$ and $\tau \in S_{\lambda}$. By a theorem of Mañé, Sad and Sullivan [16], $E^{\tau \lambda}: \mathbb{C} \rightarrow \mathbb{C}$ is quasiconformal, for a fixed $\tau \in S_{\lambda}$. Let $\mu_{\tau}$ be the Beltrami coefficient of $E^{\tau \lambda}$ in the upper half plane $\mathbb{H}^{2}$. Then $\tau \mapsto\left[\mu_{\tau}\right]$ is a holomorphic map from $S_{\lambda}$ to $\mathcal{T}\left(\mathbb{H}^{2}\right)$, where $\left[\mu_{\tau}\right]$ is the Teichmüller class of $\mu_{\tau}$ in $\mathcal{T}\left(\mathbb{H}^{2}\right)$. This map is called a complex earthquake and it agrees with McMullen's complex earthquake definition for finite surfaces with small imaginary part of the parameter.

Corollary 1. Given a bounded measured lamination $\lambda$ in $\mathbb{H}^{2}$ there is a well-defined complex earthquake $\tau \mapsto\left[\mu_{\tau}\right]$ for all parameters $\tau$ in a neighborhood $S_{\lambda}$ of the real line $\mathbb{R}$. The complex earthquake is holomorphic and it extends the earthquake path $t \mapsto\left[\left.E^{t \lambda}\right|_{\widehat{\mathbb{R}}}\right]$. Consequently, the earthquake path is real analytic in the parameter.

Because we work with the universal Teichmüller space $\mathcal{T}\left(\mathbb{H}^{2}\right)$ our results are more general than previous results 12, 14, 17. They hold for Teichmüller spaces of arbitrary Riemann surfaces. They show that if we bend a small amount the bent surface is convex (a result previously known for measured laminations of finite surfaces [12]). Our results extend Kerckhoff's result on analyticity of earthquake paths for finite surfaces and McMullen's result of holomorphicity of complex earthquakes for finite surfaces to arbitrary hyperbolic surfaces.

There is a significant difference in techniques used for finite surfaces case and for arbitrary surfaces case. Mainly, in the finite case the role of closed geodesics is distinguished and they are used to approximate general laminations. In the infinite case, we are forced to work in the hyperbolic plane where no closed geodesics exist and their role is played by laminations with a finite number of leaves.

The paper is organized as follows. In Section 2 we define earthquakes and earthquake measures, show that bounded measures correspond to earthquakes, and give one direction of the proof of Theorem 1. In Section 3 we review the definition of bending. In Section 4 we recall some theorems on holomorphic motions. Using Theorem 2 and holomorphic motions we prove Corollary 1. Corollary 1 directly proves the second direction of Theorem 1. In Section 5 we give the main geometric argument in this paper. It states that if the parameter has small imaginary part, then the image of a geodesic ray under the bending is contained in a cone of a fixed angle with vertex the initial point of the ray. In Section 6 we use the cone argument to prove Theorem 2.

\section{EARThquAKES AND EARTHQUAKE MEASURES}

We define earthquakes and investigate their properties. All our discussions are for the hyperbolic plane $\mathbb{H}^{2}$. The general theory for complete hyperbolic surfaces follows from the fact that $\mathbb{H}^{2}$ is the universal covering of any complete hyperbolic surface 19 . We begin with the definition of a geodesic lamination on $\mathbb{H}^{2}$.

Definition 2.1. A geodesic lamination $\mathcal{L}$ on $\mathbb{H}^{2}$ is a closed subset of $\mathbb{H}^{2}$ that is written as a disjoint union of geodesics in $\mathbb{H}^{2}$. A flat piece of $\mathcal{L}$ is a connected component of the complement of $\mathcal{L}$ in $\mathbb{H}^{2}$. A facet of $\mathcal{L}$ is either a geodesic of $\mathcal{L}$ or a flat piece. The union of all facets of $\mathcal{L}$ covers $\mathbb{H}^{2}$.

The union of all simple geodesics on a finite area hyperbolic surface has Hausdorff dimension 1. In particular, the Lebesgue measure of a geodesic lamination on a 
finite surface is zero. Also, a geodesic lamination on a finite surface can be written in a unique way as a disjoint union of geodesics.

For $\mathbb{H}^{2}$ this is not true. In fact, a geodesic lamination $\mathcal{L}$ on $\mathbb{H}^{2}$ can fill the space. For this reason we require that the decomposition of $\mathcal{L}$ into a disjoint union of geodesics is a part of the above definition.

Definition 2.2. A measured lamination $\lambda$ on $\mathbb{H}^{2}$ is given by the support geodesic lamination $|\lambda|$ and by the transverse measure to $|\lambda|$. In more detail, we assign a finite Borel measure to each closed finite length geodesic arc in $\mathbb{H}^{2}$ whose support is on the transverse intersection of the arc with $|\lambda|$. The measure on arcs is required to be invariant under homotopies preserving geodesics of $|\lambda|$.

We allow for positive, real or complex measured laminations depending on our needs. Following Thurston [19], we give the definition of an earthquake.

Definition 2.3. An earthquake $E$ is a bijective map of $\mathbb{H}^{2}$ onto itself with the following properties: The support of the earthquake is a geodesic lamination $\mathcal{L}$. On each facet of $\mathcal{L}$, the earthquake $E$ is a Möbius map. For any two facets $A$ and $B$, the map $\left.E\right|_{B} \circ\left(\left.E\right|_{A}\right)^{-1}$ is a hyperbolic translation with axis separating $A$ and $B$. Further, $\left.E\right|_{B} \circ\left(\left.E\right|_{A}\right)^{-1}$ translates to the left as seen from $A$.

An earthquake is not necessarily a homeomorphism of $\mathbb{H}^{2}$. Thurston [19] showed that an earthquake continuously extends to an orientation preserving homeomorphism of the boundary $\partial_{\infty} \mathbb{H}^{2}=S^{1}$ of $\mathbb{H}^{2}$. Conversely, any orientation preserving homeomorphism of the unit circle $S^{1}$ can be obtained by continuous extension of an earthquake of $\mathbb{H}^{2}$ (geology is transitive). We use the upper half-plane model for $\mathbb{H}^{2}$ and denote its boundary $\widehat{\mathbb{R}}$ by $S^{1}$, where $\widehat{\mathbb{R}}=\mathbb{R} \cup\{\infty\}$.

If the support of an earthquake $E$ is a lamination $\mathcal{L}$ with finitely many geodesics, then it is called a finite earthquake. Let $l$ be a geodesic of $\mathcal{L}$, and let $A$ and $B$ be flat pieces adjacent to $l$. Then $\left.E\right|_{B} \circ\left(\left.E\right|_{A}\right)^{-1}$ has to be a hyperbolic translation with the axis $l$. We assign the translation length of $\left.E\right|_{B} \circ\left(\left.E\right|_{A}\right)^{-1}$ to $l$ and obtain a positive measured lamination $\lambda$ with support $\mathcal{L}$. Conversely, any positive finite measured lamination $\lambda$ corresponds to a unique finite earthquake up to some ambiguities in the definition on isolated geodesics in $|\lambda|$ and more importantly up to postcomposition with a Möbius map [19].

Given a general earthquake $E$, there is an associated positive measured lamination $\lambda$ as follows. The support of $\lambda$ equals the support of $E$. Let $I$ be a closed geodesic arc transversely intersecting the support of $E$ with arbitrary orientation. For given $n$, choose facets $\mathcal{A}_{n}=\left\{A_{1}, A_{2}, \ldots, A_{n}\right\}$ of the support of $E$ such that $A_{1}$ contains the left endpoint of $I$ and $A_{n}$ contains the right endpoint of $I$, and $A_{i}$ intersects $I$ in the given order. The sum of the translation lengths of $\left.E\right|_{A_{i+1}} \circ\left(\left.E\right|_{A_{i}}\right)^{-1}$ for $i=1,2, \ldots, n-1$ is the approximate measure of $I$. If $n \rightarrow \infty$ and $\mathcal{A}_{n}$ are chosen such that the union of their elements is dense in $I$, then the limit of approximate measures for $\mathcal{A}_{n}$ is a well-defined measure 19. If the endpoints of $I$ are not in the interior of flat pieces some additional care in the definition is needed. The measure of a point is the limit of measures of closed geodesic arcs transverse to the support of $E$ which contain the point in their interiors, and which descend to the point. If the left endpoint $a$ of $I$ is not on the flat piece of the support of $E$, then we choose $\mathcal{A}_{n}$ such that $A_{1}$ does not contain $a$ but it is close to $a$ for $n$ large. The mass of $I$ is the limit of approximate measures for $\mathcal{A}_{n}$ plus half of the mass for $a$. Similarly for the right endpoint of $I$ if it does not belong to a flat piece. 
We call the associated positive measured lamination an earthquake measure. Thurston [19] showed that earthquakes are uniquelly determined up to post-composition with a Möbius map by the associated earthquake measure.

More generally, a positive measured lamination on $\mathbb{H}^{2}$ is called an earthquake measure. An earthquake measure does not always give an earthquake; see examples in [20] and in [10]. In general, for a given earthquake measure $\lambda$ there is a cocycle map $E^{\lambda}$ from $\mathbb{H}^{2}$ to $\mathbb{H}^{2}$ which satisfies all the properties of an earthquake except possibly being onto; see Epstein-Marden [5. The cocyle for a given measure is constructed by taking the limit of finite earthquakes corresponding to finite measure approximation to the earthquake measure. More details on the construction of cocycles for (complex) measured laminations is given in Section 3. A natural question is which measures give an earthquake map. We give a sufficient condition which is not necessary.

Definition 2.4. An earthquake measure $\lambda$ is bounded if $\sup \lambda(I)$ is finite, where the supremum is over all unit length closed geodesic arcs in $\mathbb{H}^{2}$.

We give a lower bound estimate in terms of an earthquake measure $\lambda$ on the distance between the images under the corresponding cocycle map $E^{\lambda}$ for two geodesics of $|\lambda|$, given their original distance.

Lemma 2.1. Let $\lambda$ be an earthquake measure on $\mathbb{H}^{2}$ and let $E^{\lambda}$ be the corresponding cocycle map. Let $l_{1}$ and $l_{2}$ be two geodesics of $|\lambda|$ whose distance is $d$. The distance $d_{1}$ between $E^{\lambda}\left(l_{1}\right)$ and $E^{\lambda}\left(l_{2}\right)$ satisfies

$$
d_{1} \geq C(d) e^{-\lambda(I)},
$$

where $C(d)=\frac{d}{\cosh d+1}$ and $\lambda(I)$ is the measure of a closed arc $I$ which connects $l_{1}$ and $l_{2}$.

Proof. The hyperbolic metric on $\mathbb{H}^{2}$ is invariant under Möbius maps. Thus without loss of generality, we can assume that $l_{1}$ has endpoints 0 and $\infty$, and that $l_{2}$ has endpoints 1 and $c, c>1$. We normalize $E^{\lambda}$ by post-composing it with a Möbius map, if necessary, to be the identity on $l_{1}$. Then $E^{\lambda}\left(l_{1}\right)=l_{1}$.

The cocycle map $E^{\lambda}$ is obtained by taking the limit of finite compositions of hyperbolic translations with axis between $l_{1}$ and $l_{2}$ oriented to the left as seen from $l_{1}$. It is obvious that $E^{\lambda}\left(l_{2}\right)$ will be the closest to $l_{1}$ if all hyperbolic translations in the composition have the repelling fixed point 1 and the attracting fixed point $\infty$. In this case $E^{\lambda}\left(l_{2}\right)$ is the geodesic with endpoints 1 and $M c$, where $M \leq e^{\lambda(I)}$.

A computation in the hyperbolic plane $\mathbb{H}^{2}$ gives $\cosh d=\frac{c+1}{c-1}$ and $\cosh d_{1}=$ $\frac{M c+1}{M c-1}$. Then

$$
d_{1} \geq \log \cosh d_{1}=\log \frac{M c+1}{M c-1} \geq \frac{1}{M c}
$$

and

$$
\frac{1}{c}=\frac{\cosh d-1}{\cosh d+1} \geq \frac{d}{\cosh d+1}=C(d) .
$$

We combine the above inequalities to obtain (11).

We show that a bounded earthquake measure gives a cocyle map which is an earthquake. This condition is not necessary. 
Proposition 2.1. Let $\lambda$ be a bounded earthquake measure. Then there exists an earthquake $E^{\lambda}$ whose measure is $\lambda$.

Proof. As remarked above, we only need to show that $E^{\lambda}: \mathbb{H}^{2} \rightarrow \mathbb{H}^{2}$ is onto.

The union of facets of $|\lambda|$ covers $\mathbb{H}^{2}$. The images of facets of $|\lambda|$ under $E^{\lambda}$ are mapped into $\mathbb{H}^{2}$ and they inherit separation properties from $|\lambda|$. Assume $\left\{l_{i}\right\}$ is a sequence of geodesics in $|\lambda|$ and $\left\{P_{i}\right\}$ is a sequence of half-planes with boundary $l_{i}$ such that $P_{i+1} \subset P_{i}$ and that the intersection of the closures of $P_{i}$ in $\mathbb{H}^{2} \cup S^{1}$, for all $i$, is a unique point on $S^{1}$. It is enough to show that for any such sequence $\left\{l_{i}\right\}$, the sequence $\left\{E^{\lambda}\left(l_{i}\right)\right\}$ satisfies the same property. There are two possibilities for $\left\{l_{i}\right\}$. Either all but finitely many geodesics $l_{i}$ have a common endpoint or there is a subsequence, denoted by $\left\{l_{i}\right\}$ again, such that consecutive pairs $l_{i}$ and $l_{i+1}$ are a positive distance apart.

Assume that all $l_{i}$ have a common endpoint $x \in \widehat{\mathbb{R}}$. Transverse measure $\lambda$ can be considered as a measure on the space of geodesics of $\mathbb{H}^{2}$. We use this description simultaneously with the transverse description for the simplicity of notation. Since $l_{1}$ and $l_{i}$ are distance 0 apart and since $\lambda$ is bounded, we conclude that the mass, for the $\lambda$ measure, of all geodesics of $|\lambda|$ which have one endpoint $x$ is finite. Without loss of generality, we can assume that $l_{i}$ has endpoints $\infty$ and $x_{i}<0$, and $x_{i}>x_{i+1}$, and $x_{i} \rightarrow-\infty$ as $i \rightarrow \infty$. Let $E^{\lambda}$ be the identity on the geodesic with endpoints 0 and $\infty$. Then $\left.E^{\lambda}\right|_{l_{i}}$ is the limit of finite compositions of hyperbolic translations with axis between $l_{i-1}$ and $l_{i}$, and whose total translation length is $\lambda\left(I_{i}\right)=a_{i}$, where $I_{i}$ is a closed geodesic arc connecting $l_{i-1}$ and $l_{i}$. By the above $\sum_{i=1}^{\infty} a_{i}=$ $a<\infty$. Then $E^{\lambda}$ fixes $\infty$ and moves $x_{i}$ toward 0 . An easy observation shows that $E^{\lambda}\left(x_{i}\right) \leq e^{-a} x_{i} \rightarrow-\infty$ as $i \rightarrow \infty$. Consequently, $E^{\lambda}\left(x_{i}\right) \rightarrow-\infty$ as $i \rightarrow \infty$ which proves the claim in this case.

Assume that we are in the second case. Namely, there exists a nested sequence $\left\{l_{i}\right\}$ of geodesics of $|\lambda|$ with the following properties. The hyperbolic plane $\mathbb{H}^{2}$ is divided into two hyperbolic half-planes by each $l_{i}$. Let $P_{i}$ be the hyperbolic halfplane which contains $l_{i+1}$. The intersection of closures of $P_{i}$ in $\mathbb{H}^{2} \cup S^{1}$ equals a point $x \in S^{1}$. By choosing a possibly different sequence $\left\{l_{i}\right\}$ of $|\lambda|$ we arrange that the distance between all consecutive $l_{i}$ and $l_{i+1}$ lies in the interval [1,2] as follows. If the distance between two consecutive geodesics $l_{i}$ and $l_{i+1}$ is larger than 2 , then we add to the sequence geodesics with zero mass in between $l_{i}$ and $l_{i+1}$. If the distance between $l_{i}$ and $l_{i+1}$ is less than 1 , then there exists $l_{j}, j>i+1$, such that the distance between $l_{i}$ and $l_{j}$ is greater than or equal to 1 . Then we replace $l_{i+1}$ by $l_{j}$ and proceed as above. It remains to show that for each $l_{i}$ there exists $l_{j}$, $j>i$, such that their distance is greater than or equal to 1 . If not, there are two possibilities. Either the distance between $l_{j}$ and $l_{i}$ is 0 for all $j>i$ or there is $l_{j}$, for some $j>i$, such that the distance between $l_{j}$ and $l_{i}$ is greater than 0 . The first possibility is in the contradiction with the fact that $x$ is not an endpoint of $l_{i}$. If the second possibility holds, then the set $l_{k}, k>j$, is compact because $l_{j}$ separates $l_{i}$ and $l_{k}$, the distance between $l_{j}$ and $l_{i}$ is greater than 0 , and the distance between $l_{i}$ and $l_{k}$ is less than 1 . Consequently, $l_{k}$ do not accumulate on $x \in \widehat{\mathbb{R}}$ which gives a contradiction.

It is enough to show that the distance between the images of $l_{1}$ and $l_{i}$ under $E^{\lambda}$ goes to infinity as $i \rightarrow \infty$. The distance between $l_{1}$ and $l_{i}$ is greater than the sum of distances between $l_{j}$ and $l_{j+1}$ for $j=1,2, \ldots, i-1$. Let $I_{j}$ be a closed geodesic arc connecting $l_{j}$ and $l_{j+1}$, and let $d_{j}$ equal the distance between $l_{j}$ and $l_{j+1}$. By Lemma 
2.1 , the above sum is bounded below by $\sum_{j=1}^{i-1} e^{-\lambda\left(I_{j}\right)} C\left(d_{j}\right) \geq \bar{C} e^{-2\|\lambda\|}(i-1) \rightarrow \infty$ as $i \rightarrow \infty$. The constant $\bar{C}>0$ exists because $C(d)$ is a positive continuous function for $d>0$ and $d_{j} \in[1,2]$. This establishes the proposition.

We prove that bounded earthquake measures give quasisymmetric maps and conversely, quasisymmetric maps determine earthquakes with bounded measures. This allows us to consider earthquakes as elements of the universal Teichmüller space $\mathcal{T}\left(\mathbb{H}^{2}\right)$.

Proof of Theorem 1. Let $\left.E^{\lambda}\right|_{S^{1}}$ be quasisymmetric and assume on the contrary that $\lambda$ is not bounded. Thus, there exists a sequence of closed geodesic arcs $I_{n}$ such that the length of $I_{n}$ converges to 0 and $\lambda\left(I_{n}\right) \rightarrow \infty$ as $n \rightarrow \infty$. This follows by the use of Cantor diagonalization process.

Choose an arbitrary orientation of $I_{n}$. Let $l_{1}^{n}$ and $l_{2}^{n}$ be geodesics of $|\lambda|$ which intersect $I_{n}$ closest to or at its left and right endpoints, respectively. Post-compose $E^{\lambda}$ with a Möbius map such that its restriction to $l_{1}^{n}$ is the identity and call it $E^{\lambda}$ again. We conjugate $E^{\lambda}$ with a Möbius map $\gamma_{n}$ such that $\gamma_{n}\left(l_{1}^{n}\right)$ is the geodesic with endpoints 0 and $\infty$, and $\gamma_{n}\left(l_{2}^{n}\right)$ is the geodesic with endpoints 1 and $c_{n}, c_{n}>1$. We obtain a new earthquake $E^{n}=\gamma_{n} \circ E^{\lambda} \circ \gamma_{n}^{-1}$. Let $f$ be a quasiconformal extension of $\left.E^{\lambda}\right|_{S^{1}}$ to $\mathbb{H}^{2}$. Then $f_{n}=\gamma_{n} \circ f \circ \gamma_{n}^{-1}$ is a quasiconformal extension of $\left.E^{n}\right|_{S^{1}}=\left.\gamma_{n} \circ E^{\lambda}\right|_{S^{1}} \circ \gamma_{n}^{-1}$ to $\mathbb{H}^{2}$. The quasiconformal constant of $f_{n}$ is equal to the quasiconformal constant of $f$. Consequently, $\left.f_{n}\right|_{S^{1}}$ is quasisymmetric with the same constant as $\left.f\right|_{S^{1}}$ and each $E^{n}$ is the identity on the geodesic with endpoints 0 and $\infty$. Thus $f_{n}(\infty)=\infty$ and $f_{n}$ restricts to a map of $\mathbb{R}$ onto itself.

We prove that the quasisymmetric constant of $f_{n}: \mathbb{R} \rightarrow \mathbb{R}$ tends to infinity as $n \rightarrow \infty$ which is a contradiction to the above; see [1]. By our normalization, $\gamma_{n}\left(l_{2}^{n}\right)$ has fixed points 1 and $c_{n}$ such that $c_{n} \rightarrow \infty$ as $n \rightarrow \infty$. Clearly, $f_{n}(0)=0$ and $-2 \leq f_{n}(-2)<0$ because $E^{n}$ is a left earthquake. Since $\lambda\left(I_{n}\right) \rightarrow \infty$, it is also true that $f_{n}(2) \rightarrow \infty$ as $n \rightarrow \infty$. Thus the ratio

$$
\frac{f_{n}(2)-f_{n}(0)}{f_{n}(0)-f_{n}(-2)} \rightarrow \infty
$$

as $n \rightarrow \infty$. This shows that the constant of quasisymmetry of $f_{n}$ tends to infinity. The contradiction proves that $\lambda$ is bounded.

To prove the opposite direction we assume that $\lambda$ is bounded. In Proposition 1 , we showed that $E^{\lambda}$ is an earthquake. It remains to show that $\left.E^{\lambda}\right|_{S^{1}}$ is quasisymmetric. A direct proof of this fact is certainly possible. However, Theorem 2 and the properties of holomorphic motions guarantee that $\left.E^{\lambda}\right|_{S^{1}}$ is quasisymmetric. Thus the proof will be complete once we prove Theorem 2 . Note that we do not use the fact that $\left.E^{\lambda}\right|_{S^{1}}$ is quasisymmetric in the proof of Theorem 2.

Let $\lambda$ be a bounded earthquake measure. The corresponding earthquake $E^{\lambda}$ when restricted to $S^{1}$ is a quasisymmetric map. An earthquake measure corresponds to the class of quasisymmetric maps where two maps are equivalent if they differ by a post composition with a Möbius map. Our interest is $\mathcal{T}\left(\mathbb{H}^{2}\right)$ and we do not distinguish between equivalent quasisymmetric maps. Consequently to each earthquake measure there corresponds a unique point of $\mathcal{T}\left(\mathbb{H}^{2}\right)$. By abuse of notation, we say that two earthquakes are the same if their continuous extensions to $S^{1}$ differ by the post-composition with a Möbius map. Multiplying $\lambda$ with a positive 
parameter $t$, we obtain a path of bounded earthquake measures. The corresponding earthquakes $E^{t \lambda}$ form an earthquake path $t \mapsto\left[\left.E^{t \lambda}\right|_{S^{1}}\right]$ in $\mathcal{T}\left(\mathbb{H}^{2}\right)$.

We note that for an arbitrary hyperbolic surface $X$, there is an injective correspondence between covering group invariant earthquake measures and covering group invariant quasisymmetric maps of $S^{1}$. Thus we obtain an earthquake path $t \mapsto\left[\left.E^{t \lambda}\right|_{S^{1}}\right]$ in $\mathcal{T}(X)$

\section{Bending measure AND COCYCle MAP}

We extend the positive parameter $t$ for earthquake path $t \mapsto\left[\left.E^{t \lambda}\right|_{S^{1}}\right]$ into a complex parameter $\tau$. For an earthquake measure $\lambda$ and a complex parameter $\tau \in \mathbb{C}, \tau \lambda$ is a complex measured lamination of special form (positive measure times complex number). We consider a general complex measured lamination.

Definition 3.1. A bending measure $\lambda$ is a complex Borel transverse measure to a fixed geodesic lamination $|\lambda|$, called the support of $\lambda$.

Thurston introduced bending cocycle $E^{\lambda}: \mathbb{H}^{2} \rightarrow \mathbb{H}^{3}$ for the bending measure $\lambda$. Following Epstein-Marden [5] we take the upper half-space model $\{(z, t): z \in$ $\mathbb{C}, t>0\}$ of the hyperbolic 3 -space $\mathbb{H}^{3}$. The hyperbolic plane $\mathbb{H}^{2}$ is embedded in $\mathbb{H}^{3}$ as the euclidean half-plane orthogonal to the $x$-axis in $\mathbb{C}$. The boundary of $\mathbb{H}^{3}$ is $\widehat{\mathbb{C}}=\mathbb{C} \cup\{\infty\}$ and the boundary of $\mathbb{H}^{2}$ is $\widehat{\mathbb{R}}=\mathbb{R} \cup\{\infty\}$.

Denote by $T_{l^{*}}^{a}$ a hyperbolic isometry of $\mathbb{H}^{3}$ which is obtained by extending the action of

$$
\left(\begin{array}{cc}
e^{\frac{a}{2}} & 0 \\
0 & e^{-\frac{a}{2}}
\end{array}\right) \in S L(2, \mathbb{C})
$$

on $\widehat{\mathbb{C}}$, where $l^{*}$ denotes the hyperbolic geodesics in $\mathbb{H}^{3}$ with endpoints 0 and $\infty$, and $a$ is the translation length. For any oriented geodesic $l$, denote by $M \in S L(2, \mathbb{C})$ a hyperbolic isometry which maps the initial point of $l$ to 0 and the terminal point of $l$ to $\infty$. Define $T_{l}^{a}=M^{-1} \circ T_{l^{*}}^{a} \circ M$.

Assume that $|\lambda|$ is finite. Then $\lambda$ is given by an assignment of complex numbers to each geodesic of $|\lambda|$. Let $A$ be a fixed flat piece of $|\lambda|$. We define $\left.E^{\lambda}\right|_{A}=i d$. Let $B$ be any other flat piece. We connect $A$ to $B$ by a geodesic $\operatorname{arc} c$. Let $l_{0}, l_{1}, \ldots, l_{n}$ be geodesics of $|\lambda|$ which intersect $c$ in the given order from $A$ to $B$. We orient them to the left as seen from $A$. Let $a_{0}=\lambda\left(l_{0}\right), a_{1}=\lambda\left(l_{1}\right), \ldots, a_{n}=\lambda\left(l_{n}\right)$. Let $A_{0}=A, A_{1}, \ldots, A_{n}, A_{n+1}=B$ be flat pieces along $c$ in the given order. Define a bending cocycle $\left.E^{\lambda}\right|_{B}=T_{l_{0}}^{a_{0}} \circ T_{l_{1}}^{a_{1}} \circ \cdots \circ T_{l_{n}}^{a_{n}}$ and $\left.E^{\lambda}\right|_{l_{n}}=T_{l_{0}}^{a_{0}} \circ T_{l_{1}}^{a_{1}} \circ \cdots \circ T_{l_{n}}^{\frac{a_{n}}{2}}$. We consider $E^{\lambda}$ and $M \circ E^{\lambda}$, where $M$ is a Möbius map, as equivalent cocycles and do not distinguish between them.

Let

$$
M=\left(\begin{array}{ll}
a & b \\
c & d
\end{array}\right) \in S L(2, \mathbb{C})
$$

Define the norm of $M$ by $\|M\|=\max \{|a|+|b|,|c|+|d|\}$.

Assume that $\lambda$ is an arbitrary bending measure. Then $|\lambda|$ could fill out $\mathbb{H}^{2}$. We fix a facet $A$ of $|\lambda|$ such that $A$ is either a flat piece or a geodesic of $|\lambda|$ with $\lambda(A)=0$. Define $\left.E^{\lambda}\right|_{A}=i d$. Let $B$ be an arbitrary facet of $|\lambda|$ and let $c$ be a geodesic arc connecting $A$ to $B$.

We choose a sequence of finite approximations to $\lambda$ on the part which intersects $c$ as follows. Fix $n$ and choose points $C_{0}, C_{1}, \ldots, C_{k(n)}$ on $c$ with the following properties. Points $C_{0}$ and $C_{k}$ are the endpoints of $c$, the distance between $C_{i-1}$ 
and $C_{i}$ is less than $\frac{1}{n}$, and $\lambda\left(C_{i}\right)=0$ for $i=1,2, \ldots, k-1$. If $\lambda\left(\left[C_{i-1}, C_{i}\right]\right) \neq 0$ for $i=1,2, \ldots, k-1$, then we choose one geodesic of $|\lambda|$ which intersects $\left[C_{i-1}, C_{i}\right]$ and assign to it weight $\lambda\left(\left[C_{i-1}, C_{i}\right]\right)$. If $\lambda\left(\left[C_{k-1}, C_{k}\right]\right) \neq 0$, then we choose one geodesic which intersects $\left[C_{k-1}, C_{k}\right]$ and assign to it weight $\lambda\left(\left[C_{k-1}, C_{k}\right)\right)+\frac{1}{2} \lambda\left(C_{k}\right)$. Similarly for $\lambda\left(\left[C_{0}, C_{1}\right]\right) \neq 0$. We constructed a finite approximation $\lambda_{n}$ of $\lambda$. Let $E^{\lambda_{n}}$ be a bending cocycle for $\lambda_{n}$ which is the identity on the facet containing $C_{0}$.

Epstein-Marden [5] showed that $\left.E^{\lambda_{n}}\right|_{B}$ converges in the norm as $n$ tends to infinity. The convergence is independent of the choice of a sequence. We define $\left.E^{\lambda}\right|_{B}=\left.\lim _{n \rightarrow \infty} E^{\lambda_{n}}\right|_{B}$

\section{Holomorphic MOTiONS}

We describe needed results on holomorphic motions and apply them to our situation. To begin, we give the definition of a holomorphic motion of a closed subset of $\mathbb{C}$. Let $\Delta$ be the open unit disk and let $\Lambda$ be a fixed closed subset of $\mathbb{C}$.

Definition 4.1. A map $F: \Delta \times \Lambda \rightarrow \mathbb{C}$, given by $F(\tau, z)=f_{\tau}(z)$ for $\tau \in \Delta$ and $z \in \Lambda$, is a holomorphic motion of $\Lambda$ in $\mathbb{C}$ if the following are satisfied:

1. $f_{0}(z)=z$ for all $z$ in $\Lambda$,

2. for a fixed $\tau \in \Delta$, the map $z \mapsto f_{\tau}(z)$ is injective on $\Lambda$,

3. for a fixed $z \in \Lambda$, the map $\tau \mapsto f_{\tau}(z)$ is holomorphic in $\Delta$.

Note that in the above definition we require that the parameter for a holomorphic motion is in the unit disk $\Delta$. In Theorem 2, the parameter is not in the unit disk but in a neighborhood $S_{\lambda}$ of the real line. By pre-composition of $F$ with a Riemann mapping for $S_{\lambda}$ which maps 0 to 0 , we can arrange that the parameter is in $\Delta$. Thus we do not have to require this in the definition.

When $\tau=0$ in the situation in Theorem 2, the earthquake with zero measure $E^{0 \lambda}=E^{0}$ is the identity on $\mathbb{R}$. Thus to show Theorem 2 , we need to show that the bending cocyle extends to an injective map on $\mathbb{R}$, for a fixed parameter $\tau$, and that the extension is a holomorphic map in the parameter $\tau \in \Delta$, for a fixed $x \in \mathbb{R}$. We give the proof of these properties in the next two sections. In the rest of this section we prove Corollary 1.

Proof of Corollary 1. Slodkowski [18] showed that any holomorphic motion of a closed set $\Lambda$ in $\mathbb{C}$ can be extended to a holomorphic motion of $\mathbb{C}$. Mañé, Sad and Sullivan [16] proved that if $f_{\tau}(z)$ is a holomorphic motion of $\mathbb{C}$, then $f_{\tau_{0}}$ is a quasiconformal map of $\mathbb{C}$, for any fixed $\tau_{0} \in \Delta$. Further, if $\mu_{\tau}$ is the Beltrami differential of $f_{\tau}$ restricted to $\mathbb{H}^{2}$, then the map $\tau \mapsto \mu_{\tau}$ from $\Delta$ into the open unit ball of $L^{\infty}\left(\mathbb{H}^{2}\right)$ is holomorphic. By taking Teichmüller class of $\mu_{\tau}$ the above map gives a holomorphic map of $\Delta$ into the universal Teichmüller space $\mathcal{T}\left(\mathbb{H}^{2}\right)$.

Note that we assume $E^{\tau \lambda}(\infty)=\infty$ and consequently $E^{\tau \lambda}$ maps $\mathbb{R}$ into $\mathbb{C}$. By Theorem 2, $(x, \tau) \mapsto E^{\tau \lambda}(x)$ is a holomorphic motion of $\mathbb{R}$ with the parameter $\tau$ in a neighborhood $S_{\lambda}$ of the real line. If we take an open simply connected neighborhood of $\mathbb{R}$ which is contained in $S_{\lambda}$, then by Slodkowski's theorem there exists an extension to the holomorphic motion of $\mathbb{C}$. Further, by a theorem of Mañé, Sad and Sullivan, the map $\tau \mapsto\left[\mu_{\tau}\right]$ is holomorphic, where $\mu_{\tau}$ is the Beltrami coefficient of the extension of the holomorphic motion restricted to the upper half-plane $\mathbb{H}^{2}$. Consequently, the restriction to the real parameter $t=R e(\tau)$ is analytic map into the Teichmüller space. Namely, real earthquake paths are real analytic paths in the Teichmüller space. 
Remark 4.1. We claim that Corollary 1 remains true for earthquake paths in Teichmüller spaces of arbitrary hyperbolic surfaces. Assume that $\lambda$ is invariant for the covering group $\Gamma$ of a hyperbolic surface $X$. Then for any $\gamma \in \Gamma$ there exists a Möbius map $\gamma_{\tau}$ such that

$$
E^{\tau \lambda}(\gamma(x))=\gamma_{\tau}\left(E^{\tau \lambda}(x)\right)
$$

for all $x \in \widehat{\mathbb{R}}$; see [5]. It is enough to show that there exists an extension of the holomorphic motion $(x, \tau) \mapsto E^{\tau \lambda}(x)$ of $\widehat{\mathbb{R}}$ to a holomorphic motion $(z, \tau) \mapsto E^{\tau \lambda}(z)$ of $\widehat{\mathbb{C}}$ which satisfies (2). This is proved by Earle-Kra-Krushkal [4] using Slodkowski's theorem.

\section{Geometry of SMall Bendings}

In this section we investigate the properties of bending cocyles when the bending measure is purely imaginary with small norm. The main result in this section describes the image of a ray under the bending cocycle as seen from the image of the initial point of the ray.

It will be convenient to consider a unit tangent bundle $T^{1} \mathbb{H}^{3}$ of $\mathbb{H}^{3}$ given in coordinates $(\zeta, v)$, where $\zeta \in \mathbb{H}^{3}$ and $v$ is a unit tangent vector at $\zeta$. The distance between two points $\left(\zeta_{1}, v_{1}\right)$ and $\left(\zeta_{2}, v_{2}\right)$ in $T^{1} \mathbb{H}^{3}$ is given by

$$
d_{T^{1} \mathbb{H}^{3}}\left(\left(\zeta_{1}, v_{1}\right),\left(\zeta_{2}, v_{2}\right)\right)=d_{\mathbb{H}^{3}}\left(\zeta_{1}, \zeta_{2}\right)+\left\|v_{1}^{\prime}-v_{2}\right\|_{T_{\zeta_{2}}^{1} \mathbb{H}^{3}},
$$

where $v_{1}^{\prime}$ is the parallel transport of $v_{1}$ at point $\zeta_{2}$ along the geodesic connecting $\zeta_{1}$ and $\zeta_{2}$.

Epstein-Marden [5] relate the distance in the norm between $A \in S L(2, \mathbb{C})$ and the identity $I \in S L(2, \mathbb{C})$ to the action of $A$ on $T^{1} \mathbb{H}^{3}$. The lemma is originally stated for $\mathbb{H}^{2}$ but the same proof works for $\mathbb{H}^{3}$.

Lemma 5.1. For a compact subset $K$ of $\mathbb{H}^{3}$, there exists a constant $C$ which satisfies the following. Let $\zeta_{0} \in K$ and $A \in S L(2, \mathbb{C})$ such that $A\left(\zeta_{0}\right) \in K$. Then

$$
\frac{\|A-I\|}{C} \leq d_{T^{1} \mathbb{H}^{3}}\left(\left(\zeta_{0}, v_{0}\right), A\left(\zeta_{0}, v_{0}\right)\right) \leq C\|A-I\|
$$

for any unit tangent vector $v_{0}$ at $\zeta_{0}$.

We consider a neighborhood of a geodesic in $\mathbb{H}^{3}$ given by all points at the distance less than a fixed constant. For each point in the neighborhood we take the sphere of unit tangent vectors and call the obtained set a crescent. More precisely,

Definition 5.1. Let $l$ be a geodesic in $\mathbb{H}^{3}$ and $d>0$ be given. A crescent of width $d$ around geodesic $l$ is

$$
\begin{aligned}
D(l ; d)= & \left\{(\zeta, v) \in T^{1} \mathbb{H}^{3} \mid \text { distance from } \zeta \text { to } l\right. \text { is } \\
& \text { less than or equal to } \left.d \text { in the hyperbolic metric of } \mathbb{H}^{3}\right\} .
\end{aligned}
$$

We estimate the action on a crescent around geodesic $l$ by an element of $S L(2, \mathbb{C})$ with axis $l$.

Lemma 5.2. Given $d>0$ and $\delta>0$ there exists a constant $M$ which satisfies the following. Let $T=T_{l}^{a} \in S L(2, \mathbb{C})$ be a hyperbolic isometry with axis $l$ and with the translation length $a,|a| \leq \delta$. Then

$$
d_{T^{1} \mathbb{H}^{3}}(p, T(p)) \leq M|a|
$$

for all $p \in D(l ; d)$. 
Proof. Since the metric $d_{T^{1} \mathbb{H}^{3}}$ is invariant under the action of elements of $S L(2, \mathbb{C})$, we can assume that $l$ is a geodesic with endpoints 0 and $\infty$, and that $p=(\zeta, t)$ with $\zeta=(z, 1) \in \mathbb{H}^{3}$. Let

$$
\begin{aligned}
B(l ; d)= & \left\{\zeta \in \mathbb{H}^{3} ; \text { distance from } \zeta \text { to } l\right. \text { is less than } \\
& \text { or equal to } \left.d \text { in the hyperbolic metric of } \mathbb{H}^{3}\right\} \subset \mathbb{H}^{3} .
\end{aligned}
$$

Note that $D(l ; d) \subset T^{1} \mathbb{H}^{3}$ is obtained by taking the unit circle of tangent vectors at each point of $B(l ; d)$. Define

$$
K=B(l ; d) \cap\left\{(z, t) ; e^{-|a|} \leq t \leq e^{|a|}\right\} .
$$

Then $\zeta=(z, 1)$ and $T(\zeta)$ are in $K$. Because of the normalization, it is clear that $\|T-I\|$ is less than or equal to a constant times $|a|$. The lemma follows by an application of Lemma 5.1.

Let $r$ be a geodesic ray which starts at $\zeta \in \mathbb{H}^{3}$ and let $\alpha$ be a real number such that $0<\alpha<\pi$. A hyperbolic cone $C(\zeta, r, \alpha)$ is the set of all $\eta \in \mathbb{H}^{3}$ such that the geodesic ray starting at $\zeta$ through $\eta$ makes an angle less than $\alpha$ with $r$. The angle is measured from 0 to $\pi$. The boundary of $C(\zeta, r, \alpha)$ consists of all $\eta \in \mathbb{H}^{3}$ such that the angle between $r$ and the geodesic ray starting at $\zeta$ through $\eta$ equals $\alpha$. The shadow of $C(\zeta, r, \alpha)$ consists of all $z \in \widehat{\mathbb{C}}$ such that the geodesic ray connecting $\zeta$ to $z$ lies in $C(\zeta, r, \alpha)$. The shadow of a hyperbolic cone is a disk in $\widehat{\mathbb{C}}$.

A point $(\zeta, v) \in T^{1} \mathbb{H}^{3}$ uniquely determines a geodesic ray $r_{v}$ starting at $\zeta$ and tangent to $v$. Conversely, a geodesic ray $r$ starting at $\zeta$ gives a point $(\zeta, v) \in T^{1} \mathbb{H}^{3}$, where $v$ is the tangent vector to $r$ at $\zeta$. Then we define $C(\zeta, v, \alpha)=C\left(\zeta, r_{v}, \alpha\right)$.

We generalize a cone argument of Keen-Series [12] to our situation. They used the geometry of cones for finite surfaces and we use a similar argument for general hyperbolic surfaces. Our goal is to prove that the image of a ray under the bending map is contained in a hyperbolic cone for small enough pure imaginary bending measure. We divide the argument into several lemmas.

The following lemma relies on elementary hyperbolic geometry.

Lemma 5.3. Given $\alpha, 0<\alpha<\pi$, and a geodesic ray $r$ starting at $\zeta \in \mathbb{H}^{3}$, there exists a number $\beta, \beta>\alpha$, such that cone $C\left(\zeta_{1}, r_{1}, \beta\right)$ is contained in $C(\zeta, r, \alpha)$, where $\zeta_{1} \in r$ is a point at the distance 1 from $\zeta$ and $r_{1}$ is a sub-ray of $r$ starting at $\zeta_{1}$. Moreover, the difference $\beta-\alpha>0$ depends only on $\alpha$.

Proof. Let $P$ be an arbitrary geodesic plane in $\mathbb{H}^{3}$ which contains $r$. Denote by $l$ one of the two geodesic rays of the boundary of $C(\zeta, r, \alpha)$ which are contained in $P$. Let $z \in \widehat{\mathbb{C}}$ be the endpoint of $l$. Form a hyperbolic triangle $T$ with vertices $\zeta$, $\zeta_{1}$ and $z$. The triangle $T$ has a zero angle at $z$. We choose $\beta$ to be equal to the exterior angle of $T$ at $\zeta_{1}$. Consequently $C\left(\zeta_{1}, r_{1}, \beta\right) \subset C(\zeta, r, \alpha)$.

By the Gauss-Bonnet theorem the sum of the the angles in $T$ is less than $\pi$, namely $\alpha+(\pi-\beta)+0<\pi$. This gives $\beta>\alpha$. The length of the finite side $\left[\zeta, \zeta_{1}\right]$ is 1. Thus, by a formula from the hyperbolic trigonometry, the difference $\beta-\alpha>0$ depends only on $\alpha$.

Let $v$ be the tangent vector at $\zeta$ to a geodesic ray $r$ with the initial point $\zeta$. Take $\zeta_{1} \in r$ at the distance 1 from $\zeta$. Denote by $r_{1}$ a sub-ray of $r$ starting at $\zeta_{1}$ and by $v_{1}$ a tangent vector to $r_{1}$ at $\zeta_{1}$. Given a bending measure $\lambda$, let $A$ and $A_{1}$ be facets of $|\lambda|$ which contain $\zeta$ and $\zeta_{1}$, respectively. Maps $\left.E^{\lambda}\right|_{A}$ and $\left.E^{\lambda}\right|_{A_{1}}$ are the 
restrictions of the bending cocycle to $A$ and $A_{1}$. Thus they are Möbius maps and we can consider their actions on $T^{1} \mathbb{H}^{3}$, not only on $A$ and $A_{1}$.

Lemma 5.4. Given $\delta>0$ there exists a constant $M$ such that for all bending measures $\lambda$ with $\|\lambda\|<\delta$,

$$
d_{T^{1} \mathbb{H}^{3}}\left(\left.E^{\lambda}\right|_{A}\left(\zeta_{1}, v_{1}\right),\left.E^{\lambda}\right|_{A_{1}}\left(\zeta_{1}, v_{1}\right)\right) \leq M\left|\lambda\left(\left[\zeta, \zeta_{1}\right]\right)\right|,
$$

where $(\zeta, v),\left(\zeta_{1}, v_{1}\right), A$ and $A_{1}$ are as above.

Proof. Without loss of generality, we assume that $\left.E^{\lambda}\right|_{A}=i d$. Then $\left.E^{\lambda}\right|_{A_{1}}$ is the limit of cocyles with finite bending measure approximations $\lambda_{n}$ of $\lambda$ along $\left[\zeta, \zeta_{1}\right]$ which are the identity on the facet containing $A$. Let $\left\{l_{1}, l_{2}, \ldots, l_{k}\right\}$ be the support of $\lambda_{n}$ and $a_{i}=\lambda_{n}\left(l_{i}\right)$. Then $\left.E^{\lambda_{n}}\right|_{A_{1}}=T_{l_{1}}^{a_{1}} \circ T_{l_{2}}^{a_{2}} \circ \cdots \circ T_{l_{k}}^{a_{k}}$ and $\left.E^{\lambda}\right|_{A_{1}}=$ $\left.\lim _{n \rightarrow \infty} E^{\lambda_{n}}\right|_{A_{1}}$.

By the invariance of the metric on $T^{1} \mathbb{H}^{3}$ under Möbius maps and using Lemma 5.2 with $d=1$, we get

$$
d_{T^{1} \mathbb{H}^{3}}\left(T_{l_{1}}^{a_{1}} \circ T_{l_{2}}^{a_{2}} \circ \cdots \circ T_{l_{i-1}}^{a_{i-1}}\left(\zeta_{1}, v_{1}\right), T_{l_{1}}^{a_{1}} \circ T_{l_{2}}^{a_{2}} \circ \cdots \circ T_{l_{i}}^{a_{i}}\left(\zeta_{1}, v_{1}\right)\right) \leq M_{1}\left|a_{i}\right|
$$

for a fixed constant $M_{1}$ and for each $i=2,3, \ldots, k$.

The triangle inequality combined with the above inequality gives

$$
d_{T^{1} \mathbb{H}^{3}}\left(\left(\zeta_{1}, v_{1}\right), E^{\lambda_{n}}\left(\zeta_{1}, v_{1}\right)\right) \leq M \sum_{i=1}^{k}\left|a_{i}\right| .
$$

Since the inequality holds for each finite approximation and $\sum_{i=1}^{k}\left|a_{i}\right| \leq 2\left|\lambda\left(\left[\zeta, \zeta_{1}\right]\right)\right|$ by the definition of $\lambda_{n}$, the lemma follows by setting $M=2 M_{1}$.

We prove the main result of this section. A purely imaginary bending measure $\lambda$ has constant sign if either $\operatorname{Im}(\lambda(I)) \geq 0$ or $\operatorname{Im}(\lambda(I)) \leq 0$ for each closed finite length geodesic $\operatorname{arc} I$.

Proposition 5.1. Given $\alpha, 0<\alpha<\pi$, there exists $\epsilon>0$ which satisfies the following. For any purely imaginary bending measure $\lambda$ of constant sign with $\|\lambda\|<$ $\epsilon$, the image under the bending cocycle $E^{\lambda}$ of a geodesic ray $r_{v}$, starting at $\zeta \in \mathbb{H}^{3}$ and tangent to $v \in T_{\zeta}^{1} \mathbb{H}^{3}$, is contained in the cone $C\left(E^{\lambda}(\zeta, v), \alpha\right)$.

Proof. We assume without loss of generality that the bending cocycle restricted to the facet containing $\zeta$ equals the identity.

Let $\zeta_{i}, i=0,1,2, \ldots$ be points on the ray $r$ in the given order such that $\zeta_{0}=\zeta$ and the distance between $\zeta_{i}$ and $\zeta_{i+1}$ equals 1 . Denote by $v_{i}$ the tangent vector to $r$ at $\zeta_{i}$ and by $A_{i}$ the facet of $|\lambda|$ which contains $\zeta_{i}$.

Then $\left.E^{\lambda}\right|_{A_{i+1}}=\left.E^{\lambda}\right|_{A_{i}} \circ E_{i}$, where $E_{i}$ is the restriction to $A_{i+1}$ of a bending cocycle for $\lambda$ normalized to be the identity on $A_{i}$. By Lemma 5.4, the distance between $\left.E^{\lambda}\right|_{A_{i+1}}\left(\zeta_{i+1}, v_{i+1}\right)$ and $\left.E^{\lambda}\right|_{A_{i}}\left(\zeta_{i+1}, v_{i+1}\right)$ is less than $M\|\lambda\|$, for all bending measures $\lambda$ such that $\|\lambda\|<\delta$.

We show that the cone $C\left(E_{i}\left(\zeta_{i+1}, v_{i+1}\right), \alpha\right)$ is inside the cone $C\left(\zeta_{i}, v_{i}, \alpha\right)$. This implies $C\left(E^{\lambda}\left(\zeta_{i+1}, v_{i+1}\right), \alpha\right) \subset C\left(E^{\lambda}\left(\zeta_{i}, v_{i}\right), \alpha\right)$, because $C\left(E_{i}\left(\zeta_{i+1}, v_{i+1}\right), \alpha\right)$ and $C\left(\zeta_{i}, v_{i}, \alpha\right)$ are mapped onto $C\left(E^{\lambda}\left(\zeta_{i+1}, v_{i+1}\right), \alpha\right)$ and $C\left(E^{\lambda}\left(\zeta_{i}, v_{i}\right), \alpha\right)$ by $\left.E^{\lambda}\right|_{A_{i}}$.

By the invariance under Möbius maps, we can assume that $\zeta_{i}=(0,0,1) \in \mathbb{H}^{3}$, $\zeta_{i+1}=\left(0,0, e^{-1}\right) \in \mathbb{H}^{3}$ and $v_{i}, v_{i+1}$ are unit tangent vectors to the oriented geodesic connecting $\infty$ to 0 at points $\zeta_{i}, \zeta_{i+1}$. The shadow of cone $C\left(\zeta_{i+1}, v_{i+1}, \beta\right)$ is a disk with center at the origin of $\mathbb{C}$. The shadow of $C\left(\zeta_{i+1}, v_{i+1}, \alpha\right)$ is contained in the 
interior of the shadow of $C\left(\zeta_{i+1}, v_{i+1}, \beta\right)$. In particular, the boundary circle $C_{\alpha}$ of the shadow of $C\left(\zeta_{i+1}, v_{i+1}, \alpha\right)$ is at the positive distance from the boundary circle $C_{\beta}$ of the shadow of $C\left(\zeta_{i+1}, v_{i+1}, \beta\right)$. Since $d_{T^{1} \mathbb{H}^{3}}\left(E_{i}\left(\zeta_{i+1}, v_{i+1}\right),\left(\zeta_{i+1}, v_{i+1}\right)\right) \leq M\|\lambda\|$, by choosing $\epsilon>0$ small enough we arrange for $E_{i}$ to be as close as we want to the identity for $\|\lambda\|<\epsilon$. Because $C_{\alpha}$ and $C_{\beta}$ are compact sets, we choose $\epsilon$ such that $E_{i}\left(C_{\alpha}\right) \cap C_{\beta}=\emptyset$. Consequently the shadow of $C\left(E_{i}\left(\zeta_{i+1}, v_{i+1}\right), \alpha\right)$ is contained in the shadow of $C\left(\zeta_{i+1}, v_{i+1}, \beta\right)$. The above inclusion, $E^{\lambda}\left(\zeta_{i+1}\right) \in C\left(\zeta_{i}, v_{i}, \alpha\right)$ and $C\left(\zeta_{i+1}, v_{i+1}, \beta\right) \subset C\left(\zeta_{i}, v_{i}, \alpha\right)$ imply

$$
C\left(E_{i}\left(\zeta_{i+1}, v_{i+1}\right), \alpha\right) \subset C\left(\zeta_{i}, v_{i}, \alpha\right)
$$

for $\epsilon$ small enough.

We prove that $E^{\lambda}\left(\left[\zeta_{i}, \zeta_{i+1}\right]\right)$ is contained in the cone $C\left(E^{\lambda}\left(\zeta_{i}, v_{i}\right), \alpha\right)$. A cone is a convex set and the endpoints of $E^{\lambda}\left(\left[\zeta_{i}, \zeta_{i+1}\right]\right)$ are in the cone $C\left(E^{\lambda}\left(\zeta_{i}, v_{i}\right), \alpha\right)$. Since the bending is in only one direction $(\lambda$ has constant sign $)$, we get $E^{\lambda}\left(\left[\zeta_{i}, \zeta_{i+1}\right]\right) \subset$ $C\left(E^{\lambda}\left(\zeta_{i}, v_{i}\right), \alpha\right)$.

Each cone $C\left(E^{\lambda}\left(\zeta_{i+1}, v_{i+1}\right), \alpha\right)$ is contained in the previous cone $C\left(E^{\lambda}\left(\zeta_{i}, v_{i}\right), \alpha\right)$ and all of them are contained in the first cone. Consequently, bent geodesic ray $E^{\lambda}(r)$ is contained in the first cone $C\left(E^{\lambda}(\zeta, v), \alpha\right)$.

Remark 5.1. The image of a ray $r$ under $E^{\lambda}$ is a bent geodesic. If $r_{i}$ is a sub-ray of $r$ with initial point $\zeta_{i}$ and with the unit tangent vector $v_{i}$, then $E^{\lambda}\left(r_{i}\right)$ is contained in cone $C\left(E^{\lambda}\left(\zeta_{i}, v_{i}\right), \alpha\right)$. The sequence of cones $C\left(E^{\lambda}\left(\zeta_{i}, v_{i}\right), \alpha\right), i=0,1,2, \ldots$, is nested. If we allow for $\lambda$ to change sign, we can still get Proposition 5.1 by further decreasing $\epsilon>0$.

A bending cocycle is defined on the boundary of facets on $S^{1}=\widehat{\mathbb{R}}$ by a simple extension. Assume that the endpoint of $r$ is not on a facet of $|\lambda|$. In order to show that the bending cocycle continuously extends to the endpoint of $r$, we could show that vertices of nested cones leave any compact subset of $\mathbb{H}^{3}$. Then the shadows of the cones would converge to a single point in $\widehat{\mathbb{C}}$. Such property is not hard to prove [12].

Instead of proving this directly we use, in the next section, properties of holomorphic maps to show that a bending cocycle map extends to all points of $S^{1}=\widehat{\mathbb{R}}$.

\section{Conclusions}

We use Proposition 5.1 to prove Theorem 2. In order to do that we divide the bending cocycle $E^{\lambda}$ into the earthquake cocycle and the pure bending cocycle.

A hyperbolic isometry $T_{l}^{a}$ with the axis $l$ and with the translation length $a=$ $\operatorname{Re}(a)+i \operatorname{Im}(a)$ can be written as a composition $T_{l}^{a}=T_{l}^{i \operatorname{Im}(a)} \circ T_{l}^{\operatorname{Re}(a)}$ of a hyperbolic translation and rotation. Assume that $\lambda$ is a finite bending measure. Let $A$ be a facet of $|\lambda|$ such that $\left.E^{\lambda}\right|_{A}=i d$. Let $B$ be any other facet of $|\lambda|$ and let $\left\{l_{1}, l_{2}, \ldots, l_{k}\right\}$ be geodesics of $|\lambda|$ which separate $A$ from $B$ in the given order. Define $a_{i}=\lambda\left(l_{i}\right)$. Then

$$
\left.E^{\lambda}\right|_{B}=T_{l_{1}}^{a_{1}} \circ T_{l_{2}}^{a_{2}} \circ \cdots \circ T_{l_{k}}^{a_{k}} .
$$

If $a, b \in \mathbb{R}$ and $l_{1}, l_{2}$ are two geodesics, then it is elementary to show that

$$
T_{l_{1}}^{a} \circ T_{l_{2}}^{i b}=T_{l_{2}^{*}}^{i b} \circ T_{l_{1}}^{a},
$$


where $l_{2}^{*}=T_{l_{1}}^{a}\left(l_{2}\right)$. We use the above to write

$$
\left.E^{\lambda}\right|_{B}=\left(T_{l_{1}^{*}}^{i I m\left(a_{1}\right)} \circ T_{l_{2}^{*}}^{i I m\left(a_{2}\right)} \circ \cdots \circ T_{l_{k}^{*}}^{i I m\left(a_{k}\right)}\right) \circ\left(T_{l_{1}}^{\operatorname{Re}\left(a_{1}\right)} \circ T_{l_{2}}^{\operatorname{Re}\left(a_{2}\right)} \circ \cdots \circ T_{l_{k}}^{\operatorname{Re}\left(a_{k}\right)}\right) \text {, }
$$

where $l_{i}^{*}=T_{l_{1}}^{\operatorname{Re}\left(a_{1}\right)} \circ T_{l_{2}}^{\operatorname{Re}\left(a_{2}\right)} \circ \cdots \circ T_{l_{i-1}}^{\operatorname{Re}\left(a_{i-1}\right)}\left(l_{i}\right)$.

If $\lambda$ is a non-finite bending measure, then $\left.E^{\lambda}\right|_{B}$ is the limit of finite bending cocycle approximation. By taking limit in the above equality, we get

$$
\left.E^{\lambda}\right|_{B}=\left.\left.E^{i \operatorname{Im}\left(\lambda^{*}\right)}\right|_{B^{\prime}} \circ E^{\operatorname{Re}(\lambda)}\right|_{B},
$$

where $\lambda^{*}=\left(E^{\operatorname{Re}(\lambda)}\right)_{*}(\lambda)$ is the push-forward of $\lambda$ by $E^{\operatorname{Re}(\lambda)}$ and $B^{\prime}=E^{\operatorname{Re}(\lambda)}(B)$.

Since $\lambda^{*}$ has different support from $\lambda$, its norm is changed. We give an upper bound estimate on the norm of $\lambda^{*}$.

Lemma 6.1. Let $\lambda$ be a bounded bending measure and let $\lambda^{*}=\left(E^{R e(\lambda)}\right)_{*}(\lambda)$ be the push-forward of $\lambda$. There exists $C>0$ such that

$$
\left\|\lambda^{*}\right\| \leq C e^{\|\lambda\|}\|\lambda\| .
$$

Proof. Let $\left\{l_{i}\right\}_{i \in \text { Ind }}$ be geodesics of $|\lambda|$ which have one endpoint in common. Then $\left|\lambda\left(\bigcup_{i \in \text { Ind }} l_{i}\right)\right|<\infty$ because $\lambda$ is a bounded measure. The push-forward geodesics $\left\{l_{i}^{*}=E^{\operatorname{Re}(\lambda)}\left(l_{i}\right)\right\}_{i \in \text { Ind }}$ of $\left|\lambda^{*}\right|$ also have one endpoint in common and

$$
\lambda^{*}\left(\bigcup_{i \in \operatorname{Ind}} l_{i}^{*}\right)=\lambda\left(\bigcup_{i \in \operatorname{Ind}} l_{i}\right)
$$

We estimate the norm of $\lambda^{*}$. Let $I^{*}$ be a closed geodesic arc of length 1 . Denote by $A^{*}$ and $B^{*}$ facets of $\left|\lambda^{*}\right|$ which contain endpoints of $I^{*}$. Let $A$ and $B$ be corresponding facets of $|\lambda|$ and take a closed geodesic arc $I$ which connects $A$ to $B$. There are two possibilities for $I$. Either all geodesics of $|\lambda|$ which intersect $I$ have one common endpoint or at least two of them are a positive distance apart.

In the first case, by (3) we get $\left|\lambda^{*}\left(I^{*}\right)\right| \leq\|\lambda\|$.

Assume that we are in the second case. Give an arbitrary orientation to $I$. Let $l$ and $r$ be geodesics of $|\lambda|$ which intersect $I$ closest to its left and right endpoints, respectively. Choose geodesic $\left\{l_{1}=l, l_{2}, \ldots, l_{k}=r\right\}$ of $|\lambda|$ intersecting $I$ in the given order and which satisfy the following. Each $l_{i}$ and $l_{i+1}$ are a positive distance apart. Denote by $I_{i}$ the closed geodesic arc orthogonal to $l_{i}$ and $l_{i+1}$. We require that the length of each $I_{i}$ is at least 1 and $\left|\lambda\left(I_{i}\right)\right| \leq 2\|\lambda\|$. Let $l_{i}^{*}=E^{\operatorname{Re}(\lambda)}\left(l_{i}\right)$ and $I_{i}^{*}$ be the closed geodesic arc orthogonal to $l_{i}^{*}$ and $l_{i+1}^{*}$.

Denote by $\operatorname{dist}\left(l^{*}, r^{*}\right)$ the distance between geodesics $l^{*}=l_{1}^{*}$ and $r^{*}=l_{k}^{*}$, and by length $\left(I_{i}^{*}\right)$ the length of a closed geodesic arc $I_{i}^{*}$. Then, by Lemma 2.1, we get

$$
\operatorname{dist}\left(l^{*}, r^{*}\right) \geq \sum_{i=1}^{k-1} \text { length }\left(I_{i}^{*}\right) \geq(k-1) C(1) e^{-\|\lambda\|},
$$

where $C(1) \geq \frac{1}{2}$ and $\operatorname{dist}\left(l^{*}, r^{*}\right) \leq 1$. Consequently, we get $k-1 \leq 2 e^{\|\lambda\|}$ because $\operatorname{dist}\left(l^{*}, r^{*}\right) \leq$ length $\left(I^{*}\right)=1$.

By the definition of push-forward

$$
\lambda^{*}\left(I^{*}\right) \leq \sum_{i=1}^{k-1} \lambda\left(I_{i}\right) \leq 2(k-1)\|\lambda\|
$$

which together with the above gives

$$
\left\|\lambda^{*}\right\| \leq 4 e^{\|\lambda\|}\|\lambda\|
$$


We are ready to prove Theorem 2 .

Proof of Theorem 2. From now on $\lambda$ is a bounded earthquake measure. In particular, it is positive. We define a neighborhood of the real line, where the complex parameter $\tau=t+i s$ will take its values.

By the previous remarks, we can write $E^{\tau \lambda}=E^{i s \lambda^{*}} \circ E^{t \lambda}$, where $\lambda^{*}=\left(E^{t \lambda}\right)_{*}(\lambda)$. Bending cocycle $E^{\tau \lambda}$ is normalized to be the identity on a facet $A$ whose boundary contains $\infty$. In particular, $E^{\tau \lambda}(\infty)=\infty$. We fix $\alpha, 0<\alpha<\frac{\pi}{2}$, and take $\epsilon>0$ from Proposition 5.1. By Lemma 6.1, we have $\left\|i s \lambda^{*}\right\| \leq|s| C e^{\|t \lambda\|}\|\lambda\|$. Define

$$
S_{\lambda}=\left\{\tau=t+i s ;|s|<\frac{\epsilon}{\left.C e^{\|t \lambda\|\|\lambda\|}\right\}}\right.
$$

We fix a point $x \in \mathbb{R}$ and define $E^{\tau \lambda}(x)$. If $x$ is on the boundary of a facet, then $E^{\tau \lambda}(x)$ is given by the cocycle and, by Epstein-Marden [5], it is holomorphic in $\tau$.

Assume that $x$ is not on a facet of $\lambda$. Then there exists a sequence of nested geodesics $l_{i}$ such that they converge to $x$. We connect a fixed facet $A$ of $\lambda$, where $\left.E^{\tau \lambda}\right|_{A}=i d$, to $E^{t \lambda}(x)=x_{t}$ by a geodesic ray $r_{t}$ with initial point $\zeta \in A$. Define an open set $V$ in $\mathbb{C}$ to consist of the union of all shadows of cones $C\left(\zeta, r_{t}, \alpha\right)$ for $t \in \mathbb{R}$. Since $0<\alpha<\frac{\pi}{2}$ the complement of $V$ in $\mathbb{C}$ contains at least three points. Let $\lambda_{n}$ be the restriction of $\lambda$ to geodesic of $|\lambda|$ which intersect $r_{0}$ between $\zeta$ and $l_{n}$. By Proposition 5.1, $E^{i s \lambda_{n}^{*}}\left(r_{t}^{n}\right)$ is contained in the cone $C\left(\zeta, r_{t}^{n}, \alpha\right)$, where $r_{t}^{n}$ is the ray with the initial point $\zeta$ and the endpoint $x_{t}^{n}=E^{\tau \lambda_{n}}(x) \in \mathbb{R}$. Thus $E^{i s \lambda_{n}^{*}}\left(x_{t}^{n}\right)$ is in $V$. For each $n$, the point $x_{t}^{n}$ is on a facet of $\lambda_{n}^{*}$ and consequently $E^{\tau \lambda_{n}}(x)$ is holomorphic in $\tau$ by [5. In addition, by the above $E^{\tau \lambda_{n}}(x) \in V$. The family of holomorphic maps $\tau \mapsto E^{\tau \lambda_{n}}(x)$ is normal by Montel's theorem and it has a convergent subsequence. For $\tau$ real, $E^{\tau \lambda}$ is an earthquake. Thus, for any two convergent subsequences, their limits agree for $\tau$ real. By the uniqueness of holomorphic maps, the limit is unique for the whole sequence and we denote it by $E^{\tau \lambda}(x)$.

It remains to show that $E^{\tau \lambda}: \mathbb{R} \rightarrow \mathbb{R}$ is injective for any $\tau \in S_{\lambda}$. This is again a consequence of Proposition 5.1. Let $x \neq y$ be two points of $\mathbb{R}$ and define $x_{1}=E^{t \lambda}(x)$ and $y_{1}=E^{t \lambda}(y)$. Then $x_{1} \neq y_{1}$ because earthquakes are injective. Take a geodesic $l$ connecting $x_{1}$ and $y_{1}$, and fix $\zeta \in l$. Let $v_{x}$ and $v_{y}$ be the unit tangent vectors to $l$ at $\zeta$ in the directions of $x_{1}$ and $y_{1}$, respectively. By Proposition 5.1 and by the above, points $E^{\tau \lambda}(x)$ and $E^{\tau \lambda}(y)$ are in the shadows of cones $C\left(E^{\tau \lambda}(\zeta), v_{x}, \alpha\right)$ and $C\left(E^{\tau \lambda}(\zeta), v_{y}, \alpha\right)$, respectively. But these shadows are disjoint since we chose $\alpha$ such that $0<\alpha<\frac{\pi}{2}$. Thus $E^{\tau \lambda}$ is injective which together with previous results finishes the proof.

Remark 6.1. By Epstein-Marden [5], the bending cocycle is holomorphic in the parameter. This allows us to claim that the bending map is holomorphic on the boundary of the facets on $S^{1}$. We showed that the bending map extends to all of $S^{1}$ and that it is holomorphic for the parameter in a neighborhood of the real line. In addition, we showed that the bending map is injective on $S^{1}$, for a fixed parameter in the above set. These two new results allowed us to use theory of holomorphic motions.

Remark 6.2. We showed that the bending cocycle extends to $\mathbb{R}$ and is holomorphic in the parameter, when the parameter is restricted to a neighborhood of the real 
line. Also, our proof shows that the extension of a bending cocycle on the whole of $\widehat{\mathbb{R}}$ is obtained as the limit of finite approximations.

Gardiner [7] gave expressions for the tangent vectors of earthquake and bending maps. The formulas are obtained by taking the limit of tangent vectors of finite approximations. Our results rigorously show that these formulas are correct.

\section{ACKNOWLEDGEMENTS}

This work was part of my dissertation. It is my great pleasure to thank Linda Keen and Frederick Gardiner for their support and advice. I am also very grateful to the referee for many suggestions which were very useful in making this paper shorter.

\section{REFERENCES}

[1] Lars V. Ahlfors, Lectures on Quasiconformal Mappings, D. Van Nostrand Company, Inc., Princeton, New Jersey, 1966. MR34:336

[2] Alan F. Beardon, The Geometry of Discrete Groups, Graduate Texts in Mathematics 91, Springer-Verlag, New York, 1983. MR 85d:22026

[3] R. D. Canary, D. B. A. Epstein, and P. Green, Notes on notes of Thurston, In D.B.A. Epstein, editor, Analytical and Geometric Aspects of Hyperbolic Space, LMS Lecture Notes 111, pages 3-92. Cambridge University Press, 1987. MR89e:57008

[4] Earle, C. J., Kra, I., Krushkal, S. L. Holomorphic motions and Teichmüller spaces, Trans. Amer. Math. Soc. 343 (1994), no. 2, 927-948. MR94h:32035

[5] D.B.A. Epstein and A. Marden, Convex hulls in hyperbolic space, a theorem of Sullivan and measured pleated surfaces, In D.B.A. Epstein, editor, Analytic and Geometric Aspects of Hyperbolic Space, LMS Lecture Notes 111, pages 112-253. Cambridge University Press, 1987. MR89c:52014

[6] D. B. A. Epstein, A. Marden and V. Markovic, Quasiconformal homeomorphisms and the convex hull boundary, Ann. of Math. 159 (2004), no. 1, 305-336. MR2052356

[7] F. Gardiner, Infinitesimal bending and twisting in one-dimensional dynamics, Trans. Amer. Math. Soc. 347 (1995), no. 3, 915-937. MR.95e:30024

[8] F. Gardiner and L. Keen, Holomorphic Motions and Quasifuchsian Manifolds, Contemp. Math. 240, 159-174, 1998. MR2000k:30023

[9] F. Gardiner and N. Lakic, Quasiconformal Teichmüller Theory, Mathematical Surveys and Monographs, Volume 76, A.M.S., 2000. MR2001d:32016

[10] F. Gardiner, J. Hu, N. Lakic, Earthquake Curves, Contemp. Math. vol. 311, 141-195, A.M.S., 2002. MR2003i:37033

[11] J. Hu, Earthquake Measure and Cross-ratio Distortion, Contemp. Math. vol. 355, 285-308, A.M.S., 2004.

[12] L. Keen and C. Series, How to bend pairs of punctured tori, In J. Dodziuk and L. Keen, editors, Lipa's Legacy, Contemp. Math. 211, 359-388, A.M.S, 1997. MR98m:30063

[13] S. Kerckhoff, The Nielsen Realization Problem, Ann. of Math. 117 (1983), 235-265. MR 85e:32029

[14] S. Kerckhoff, Earthquakes are Analytic, Comment. Math. Helv. 60 (1985), 17-30. MR86m:57014

[15] O. Lehto and K. I. Virtanen, Quasiconformal Mappings in the Plane, Second Edition, Springer-Verlag, Berlin, Heidelberg, New York, 1973. MR 49:9202

[16] R. Mañé, P. Sad and D. Sullivan, On the dynamics of rational maps, Ann. Sci. Ecole Norm. Sup, 16 (1983), 193-217. MR85j:58089

[17] C. McMullen, Complex earthquakes and Teichmuller theory, Jour. A.M.S. 11, 1998. MR 98i:32030

[18] Z. Slodkowski. Holomorphic motions and polynomial hulls, Proc. A.M.S. 111 (1991), 347-355. MR91f:58078 
[19] W. Thurston, Earthquakes in two-dimensional hyperbolic geometry. In Low-dimensional Topology and Kleinian Groups, Warwick and Durham, 1984 ed. by D.B.A. Epstein, L.M.S. Lecture Note Series 112, Cambridge University Press, Cambridge, 1986, 91-112. MR88m:57015

[20] W. Thurston, Three-Dimensional Geometry and Topology, Volume 1, Princeton University Press, Princeton, New Jersey, 1997. MR:97m:57016

Department of Mathematics, The Gradute School and University Center, The City University of New York, 365 Fifth Avenue, New York, New York 10016

Current address: Institute for Mathematical Sciences, SUNY Stony Brook, Stony Brook, New York 11794-3660

E-mail address: saric@math.sunysb.edu 